\title{
Controle in vitro de Colletotrichum truncatum do feijão fava (Phaseolus lunatus) por Trichoderma spp.
}

\author{
Otília Ricardo de Farias ${ }^{1}$, José Manoel Ferreira de Lima Cruz ${ }^{2}$, Ingrid Gomes Duarte ${ }^{3}$
}

DOI: https://doi.org/10.35699/2447-6218.2020.18464

\begin{abstract}
Resumo
A antracnose, causada por Colletotrichum truncatum é uma das principais ameaças a produção de feijão fava, com isso, medidas de controle devem ser tomadas, visando minimizar os problemas ocasionados por essa doença, como o uso de controle biológico a partir do emprego de Trichoderma spp.. Esta pesquisa objetivou-se avaliar o potencial antagônico de cepas de Trichoderma spp. sobre Colletotrichum truncatum, in vitro. Foram selecionados cinco isolados de Trichoderma spp. (TCH01, TCH02, TCH03, TCH04 e TCH05) no antagonismo a C. truncatum. Para testar o potencial antagônico das cepas de Trichoderma sobre o patógeno, foi realizado o teste de confronto direto através da metodologia de cultura pareada, no qual foi avaliado a classificação do agrupamento dos isolados do antagonista e exames microscópicos a procurando sinais de micoparasitismo. Foi realizado também o teste de antibiose, onde avaliou-se medição do diâmetro das colônias, porcentagem de inibição do crescimento micelial, taxa crescimento micelial, contagem de esporos e porcentagem de inibição da esporulação. Pelo teste de confronto direto, todos as cepas de Trichoderma spp. foram agrupados na classe 1, ou seja, os mesmos cresceram sobre a colônia do patógeno, ocupando toda placa. Também observou-se que os antagonistas possuem potencial contra $C$. truncatum no teste de antibiose, com inibição do crescimento micelial e esporulação variando de $74,7 \%$ a $82,0 \%$ e 78,7\% a 89,3\%, respectivamente. Todos as cepas avaliadas apresenta potencial antagônico sobre Colletotrichum truncatum.
\end{abstract}

Palavras-chave: Antagonismo. Antracnose. controle biológico. Phaseolus lunatus.

\section{In vitro control of broad bean Colletotrichum truncatum (Phaseolus lunatus) by Trichoderma spp.}

\begin{abstract}
Anthracnose, caused by Colletotrichum truncatum is one of the main threats to the production of fava beans, therefore, control measures must be taken, aiming to minimize the problems caused by this disease, such as the use of biological control using Trichoderma spp.. This study aimed to evaluate the antagonistic potential of the strains of Trichoderma spp. on Colletotrichum truncatum, in vitro. (TCH01, TCH02, TCH03, TCH04 and TCH05) in antagonism to C. truncatum. In order to test the antagonistic potential of Trichoderma isolates on the pathogen, the direct confrontation test was performed using the paired culture methodology, in which the classification of the cluster of the antagonist isolates and microscopic examinations looking for signs of mycoparasitism were evaluated. The antibiosis test was also carried out, in which the measurement of colony diameter, percentage of mycelial growth inhibition, mycelial growth rate, spore count and percentage of sporulation inhibition were evaluated. By the direct confrontation test, all isolates of Trichoderma spp. were grouped in class 1, that is, they grew on the pathogen colony, occupying the entire plate. It was also observed that the antagonists have potential against $C$. truncatum in the antibiosis test, with
\end{abstract}

\footnotetext{
${ }^{1}$ Universidade Federal da Paraíba. Areia, Paraíba. Brasil.

https://orcid.org/0000-0002-0753-0712

${ }^{2}$ Universidade Federal da Paraíba. Areia, Paraíba. Brasil.

https://orcid.org/0000-0002-5967-4056

${ }^{3}$ Universidade Federal Rural de Pernambuco. Recife, Pernambuco. Brasil.

https://orcid.org/0000-0002-1002-3164

*Autor para correspondência: otiliarfarias@gmail.com
}

Recebido para publicação em 25 de janeiro de 2020. Aceito para publicação em 03 de junho de 2020.

e-ISSN: 2447-6218 / ISSN: 2447-6218. Atribuição CC BY. 
de Farias, O. R. et al.

mycelial growth inhibition and sporulation ranging from $74.7 \%$ to $82.0 \%$ and $78.7 \%$ to $89.3 \%$, respectively. All the isolates evaluated were efficient antagonists for Colletotrichum truncatum.

Keywords: Antagonism. Anthracnose. Biological control. Phaseolus lunatus.

\section{Introdução}

O feijão fava (Phaseolus lunatus L.) é uma das culturas de maior importância econômica e social para a região Nordeste do Brasil, onde constitui uma importante alternativa de renda para pequenos produtores que praticam a agricultura familiar, bem como fonte alimentar para população devido ser nutricionalmente rica, principalmente em proteínas, o que a faz ser utilizado tanto na dieta humana como animal (Cavalcante et al., 2012).

Mesmo sendo uma cultura bem adaptada as condições edafoclimáticas da Região Nordeste a mesma apresenta baixa produtividade e vários são os fatores que podem estar associados a isso, como a ocorrência de doenças (Silva et al., 2014). Dentre as doenças que acometem o feijão fava no seu cultivo e que pode estar associados a baixa produtividade, a antracnose, causada pelo Colletotrichum truncatum, se destaca como uma das mais importantes (Nascimento et al., 2017).

A antracnose causa danos em folhas, ramos e vagens, e quando o ataque do patógeno é mais severo causa redução da produtividade e queda no valor dos grãos comercializados (Lopes et al., 2010), com isso, medidas de controle devem ser tomadas, para minimizar os impactos negativos desse microrganismo nos campos de cultivo.

Para doenças foliares, o controle químico é o mais utilizado, no entanto, o mesmo torna-se normalmente impraticável para antracnose do feijão fava, tendo em vista seu alto custo e por não possuir fungicidas registrados para essa doença na cultura (Henning et al., 2014). Além disso, o uso de produtos químicos vem perdendo expressão, devido impactos negativos sobre o ambiente, animais e homem, e pela procura por alimentos sem resíduos de agrotóxicos pelo mercado consumidor e surgimento de patógenos resistentes (Hillen et al., 2012).

Com isso, nos últimos anos, pesquisadores têm buscado por métodos eficientes de manejo de doenças baseados na utilização de controle biológico, a partir do emprego de Trichoderma spp. (Carvalho et al., 2011; Lazarotto et al., 2013; Pereira et al., 2014; Xue et al., 2017; Farias et al., 2019).

Trichoderma spp., compreende fungos de vida livre, que se reproduzem assexuadamente, presentes com mais frequência em solos de regiões de clima temperado e tropical (Machado et al., 2012). Esse microrganismo atua no controle de diversos fitopatógenos por diferentes mecanismos de ação, como antibiose, micoparasitismo, produção de enzimas degradadoras da parede celular, competição por nutrientes e substrato e indução de resistência (Pereira et al., 2014). Também são caracterizados por serem resistentes a substancias tóxicas produzidos por outros microrganismos e tolerantes a diferentes tipos de fungicidas (Daryaei et al., 2016), o que os tornam de elevado interesse para uso como agente de controle biológico de fitopatógenos.

Dessa maneira, estudos devem ser realizados com a finalidade de atestar o potencial antagônico desse microrganismos, com isso, a primeira etapa para avaliação da capacidade de biocontrole de Trichoderma spp. é a caracterização do potencial antagonista desses microrganismos in vitro (Mbarga et al., 2012). Vários estudos indicam que espécies de Trichoderma são agentes eficazes de biocontrole para o patógeno Colletotrichum spp. (Chargas et al., 2016; Saxena et al., 2016; Cruz-Quiroz et al., 2018; Costa et al., 2019).

Diante do exposto, objetivou-se avaliar o potencial antagônico de cepas de Trichoderma spp. sobre Colletotrichum truncatum, in vitro, responsável por danos em plantas de feijão fava (Phaseolus lunatus L.).

\section{Material e Métodos}

O experimento foi conduzido no Laboratório de Fitopatologia, do Departamento de Fitotecnia e Ciências Ambientais (DFCA), do Centro de Ciências Agrárias (CCA), na Universidade Federal da Paraíba (UFPB), Campus II, Areia, PB, Brasil.

Os isolados de Trichoderma spp. e C. truncatum utilizados nesta pesquisa pertencem à Coleção de Microrganismo do Laboratório de Fitopatologia, da Universidade Federal da Paraíba, Areia, Paraíba, Brasil. O patógeno foi obtido a partir de isolamento de plantas com sintomas de antracnose.

As cepas TCH01, TCH02, TCH03, TCH04 e TCH05 de Trichoderma spp. foram avaliados, pela técnica de confrontação direta e antibiose, no antagonismo a Colletotrichum truncatum.

Para condução experimental, culturas puras obtidas do patógeno e dos antagonistas, foram reativadas e multiplicadas em meio de cultura BDA (batata-dextrose-ágar) e mantidas em câmara de incubação por oito dias à temperatura de $25 \pm 2^{\circ} \mathrm{C}$ com fotoperíodo de 12 horas. 
Controle in vitro de Colletotrichum truncatum do feijão fava (Phaseolus lunatus) por Trichoderma spp.

O teste do confronto direto foi realizado através da metodologia de cultura pareada (Dennis; Webster, 1971). O patógeno foi repicado 3 dias antes de cada isolado do antagonista, opostamente em cada placa Petri, contendo meio de cultura BDA. As placas foram incubadas a $25 \pm 2{ }^{\circ} \mathrm{C}$, com fotoperíodo de 12 horas. O delineamento foi inteiramente casualizados, utilizando-se quatro repetições, sendo cada repetição compostas por 2 placas de Petri.

Após 7 dias da repicagem dos isolados de Trichoderma spp., foram realizadas a classificação do agrupamento dos isolados do antagonista de acordo com escala descrita por Bell et al. (1982), onde a classe 1: Trichoderma spp. cresce sobre o patógeno e ocupa toda a superfície do meio; classe 2: Trichoderma spp. cresce sobre pelo menos $2 / 3$ da superfície do meio; classe 3 : Trichoderma spp. ocupa aproximadamente metade da superfície do meio; classe 4: Trichoderma spp. cresce sobre 1/3 da superfície do meio; classe 5: Trichoderma spp. não cresce e o patógeno ocupa toda a superfície da placa.

Exames microscópicos foram feitos com aumento de 100x, procurando sinais de micoparasitismo na zona de interação entre os isolados de Trichoderma spp. e $C$. truncatum.

Para teste de antibiose, um disco da colônia do patógeno e um de cada antagonista foram adicionados individualmente no centro de placas de Petri contendo meio BDA. As placas de tamanho correspondente, contendo o Trichoderma spp. foram sobrepostas com as com C. truncatum e vedadas com plástico filme transparente e incubadas a $25 \pm 2{ }^{\circ} \mathrm{C}$, de forma que as bases superiores fossem aquelas que continham o patógeno.

Durante 7 dias foi realizada, diariamente, a medição do diâmetro das colônias de $C$. truncatum com uma régua milimetrada, em dois eixos ortogonais, descartando-se o disco repicado da colônia pura, sendo posteriormente calculada as médias. Esses dados foram utilizados no cálculo da porcentagem de inibição do crescimento micelial (PIC) e taxa crescimento micelial (Tx), com uso das seguintes fórmulas: $\mathrm{PIC}=(\mathrm{C}-\mathrm{T} / \mathrm{C}) \times 100$, onde PIC = porcentagem de inibição; $\mathrm{C}=$ crescimento radial do patógeno ( $\mathrm{mm}$ ) sozinho (controle); $\mathrm{T}=$ crescimento radial do patógeno ( $\mathrm{mm}$ ) sobre a influência dos isolados de Trichoderma spp. (Bastos, 1997). E Tx = Df/ND, sendo $\mathrm{Df}=$ diâmetro final da colônia e $\mathrm{ND}=$ números de dias de incubação

Ao final do experimento, foi feito a contagem de conídios formados ao 7ํ dia de incubação. Para tal, em cada placa de Petri foram adicionados $10 \mathrm{~mL}$ de água destilada esterilizada. A suspensão foi filtrada em dupla gaze esterilizada e retirada uma alíquota de $10 \mu \mathrm{L}$ e transferida para a câmara de Neubauer, na qual foi feita a contagem de conídios/ $\mathrm{ml}^{-1}$. Com os dados da contagem de conídios também foi calculado a porcentagem de ini- bição da esporulação (PIE) de acordo com as fórmulas apresentadas a seguir: PIE $=($ Etc-C $/$ Etc $) \times 100$, onde PIE $=$ porcentagem de inibição da esporulação; Etc = número de esporos do tratamento controle; $\mathrm{C}=$ número de esporos do patógeno sobre influência dos isolados de Trichoderma spp. (Bastos, 1997).

O delineamento experimental utilizado foi o inteiramente casualizado (DIC). As médias foram comparadas pelo teste de Scort-Knott ( $\mathrm{p}<0,05)$, utilizando software estatístico Sisvar versão 5.4 (Ferreira, 2010).

\section{Resultados e Discussão}

Todos os 5 isolados de Trichoderma spp. foram agrupados na classe 1 , ou seja, os mesmos cresceram sobre o patógeno e ocuparam toda a superfície do meio (Tabela 1). Além de colonizar eficientemente o meio, constatou-se que os mesmos produziram esporos em abundância sobre as colônias de Colletotrichum truncatum.

Tabela 1 - Classificação dos isolados de Trichoderma spp. quanto ao antagonismo exercido sobre Colletotrichum truncatum.

\begin{tabular}{cc}
\hline Tratamento & Classe* $^{*}$ \\
\hline TCH01 & 1 \\
TCH02 & 1 \\
TCH 03 & 1 \\
TCH04 & 1 \\
TCH05 & 1 \\
\hline
\end{tabular}

Segundo Mbarga et al. (2012) a primeira etapa para avaliação da capacidade de biocontrole de Trichoderma spp. é a caracterização do potencial antagonista desses microrganismos, que são realizados através de estudos in vitro. Xue et al. (2017) também sugerem que testes in vitro devem ser utilizados, principalmente, quando se deseja testar um grande número de cepas, como Trichoderma spp., que são conhecidos como micoparasitas de patógenos. E com base nos resultados iniciais obtidos nesse trabalho no teste de pareamento, observa-se que todos os isolados de Trichoderma spp. podem ser utilizados como agentes de controle biológico contra $C$. truncatum, pois os mesmos encontram-se na classe 1 , ou seja, são isolados com potencial de utilização em casa de vegetação e em campo (Tabela 1).

Nas observações microscópicas, o patógeno mostrou morfologia hifal anormal nas áreas de interação, sobre ação de todos os isolados estudados. Sendo a redução do crescimento de $C$. truncatum atribuída à competição por espaço, nutrientes presentes no meio de cultura e micoparasitismo (Vinale et al., 2008), constituindo estes como principais mecanismos utilizados pelo antagonista 
de Farias, O. R. et al.

em testes de biocontrole, como de confronto direto (Millanesi et al., 2013).

Segundo Dinesh et al. (2018), esses são uns dos mecanismos utilizados por Trichoderma spp, que atuam diretamente sobre o patógeno, principalmente através da produção de enzimas líticas, como quitinases e $\beta$-1,3-glucanases. Possuindo a capacidade de hidrolisar a parede celular do patógeno, limitando assim o seu crescimento (El_Komy et al., 2015).

Pelo teste de antibiose foi possível quantificar a produção de metabólitos voláteis pelos isolados de Trichoderma spp., com redução significativa do crescimento micelial, taxa de crescimento micelial diária e esporulação, quando comparados com a testemunha (Tabela 2).
O crescimento micelial total foi de $85,0 \mathrm{~mm}$ e a taxa de crescimento micelial diário foi de $12,1 \mathrm{~mm} \mathrm{dia}^{-1}$ na testemunha, já para as placas sobre influência dos metabolitos voláteis, o menor valor para crescimento micelial do patógeno foi com a presença do isolado TCH04, com médias de 15,3 mm e 2,1 $\mathrm{mm} \mathrm{dia}^{-1}$, respectivamente (Tabela 2).

Para esporulação, todos os isolados testados diferiram significativamente da testemunha, com destaque aos isolados TCH02, TCH03, TCHO4 e TCH05, com valores médio de 7,$5 ; 3,75 ; 6,0$ e 5,25 conídios. $\mathrm{ml}^{-1}$, respectivamente (Tabela 2 ).

Tabela 2 - Efeito inibidor de metabólitos voláteis de Trichoderma spp. sobre o crescimento micelial, taxa de crescimento micelial (TX) e esporulação (E) de Colletotrichum truncatum.

\begin{tabular}{cccc}
\hline Tratamento & $\begin{array}{c}\text { Crescimento } \\
(\mathbf{m m})\end{array}$ & $\begin{array}{c}\text { TX } \\
\left(\mathbf{m m}_{\mathbf{d i a}} \mathbf{- 1}\right)\end{array}$ & $\begin{array}{c}\text { E } \\
\left.\text { (conídios.ml }^{-1}\right)\end{array}$ \\
\hline Testemunha & $85,0 \mathrm{a}$ & $12,1 \mathrm{a}$ & $35,25 \mathrm{a}$ \\
TCH01 & $20,5 \mathrm{~b}$ & $2,9 \mathrm{~b}$ & $17,0 \mathrm{~b}$ \\
TCH02 & $21,5 \mathrm{~b}$ & $3,1 \mathrm{~b}$ & $7,5 \mathrm{c}$ \\
TCH03 & $21,1 \mathrm{~b}$ & $3,0 \mathrm{~b}$ & $3,75 \mathrm{c}$ \\
TCH04 & $15,3 \mathrm{c}$ & $2,1 \mathrm{c}$ & $6,0 \mathrm{c}$ \\
TCH05 & $19,2 \mathrm{~b}$ & $2,7 \mathrm{~b}$ & $5,25 \mathrm{c}$ \\
\hline CV $(\%)$ & 16,67 & 16,67 & 7,79 \\
\hline
\end{tabular}

Médias com letras iguais não diferem estatisticamente entre si pelo o teste de Scott Knott $(\mathrm{p}<0,05)$.

Para porcentagem de inibição do crescimento micelial, não houve diferença significativa entre os isolados de Trichoderma spp. testados, sendo observada uma redução de $74,7 \%$ a $82,0 \%$ do crescimento de $C$. truncatum, quando comparado a testemunha. Já para a inibição da esporulação, os melhores resultados foram evidenciados pelos isolados TCH02, TCH03, TCHO4 e TCH05, com valores de 78,7\%, 89,3\%, $82,9 \%$ e $85,1 \%$, respectivamente (Figura 1 ).

Os metabolitos voláteis produzidos pelo Trichoderma spp. são substâncias com ação tóxica, que possuem a capacidade de reduzir ou mesmo paralisar o crescimento e a esporulação do patógeno (Bomfim et al., 2010). O efeito dessas substâncias antifúngicas também foram relatadas por Chagas Junior et al. (2016), ao avaliar o potencial antagônico de Trichoderma spp. quanto a porcentagem de inibição do crescimento de $C$. cliviae e $C$. truncatum, onde observaram que os isolados reduziram cercas de $75 \%$ e $90 \%$ do crescimento desses patógenos, respectivamente. Fantinel et al. (2018) também observaram redução de $44,2 \%$ das colônias de $C$. siamense em função da ação inibitória dos metabolitos produzidos por Trichoderma sp.. Oliveira et al. (2016), avaliando a eficiência de isolado de Trichoderma sp. no controle de C. musae, encontraram índices de inibição de $84 \%$.

Apesar da eficiência comprovado no controle de diversos fungos fitopatogênicos, no Brasil, o controle biológico é pouco utilizado em condições de campo, devido a limitada disponibilidade de produtos comerciais à base desse fungo com registro no Ministério da Agricultura Pecuária e Abastecimento (MAPA). Apesar dos esforços de pesquisadores, a difusão de conceitos, vantagens e ações dos produtos biológicos ainda é incipiente, o que possibilita a manutenção da hegemonia do uso de produtos químicos (Machado et al., 2012). Além disso, ainda há uma carência de respostas acerca das interações entre antagonistas e o patossistemas da cultura da fava. A realização de testes em laboratório e casa de vegetação e a seleção de novos isolados de Trichoderma spp. obtidos na nossa Região, adaptados as condições do Semiárido é imprescindível na busca por novas perspectivas para emprego do controle biológico no manejo de doenças causadas por diversos patógenos em varias culturas. 
Controle in vitro de Colletotrichum truncatum do feijão fava (Phaseolus lunatus) por Trichoderma spp.

Figura 1 - Efeito inibidor de metabólitos voláteis de Trichoderma spp. sobre porcentagem de inibição do crescimento micelial e taxa de crescimento micelial de Colletotrichum truncatum.

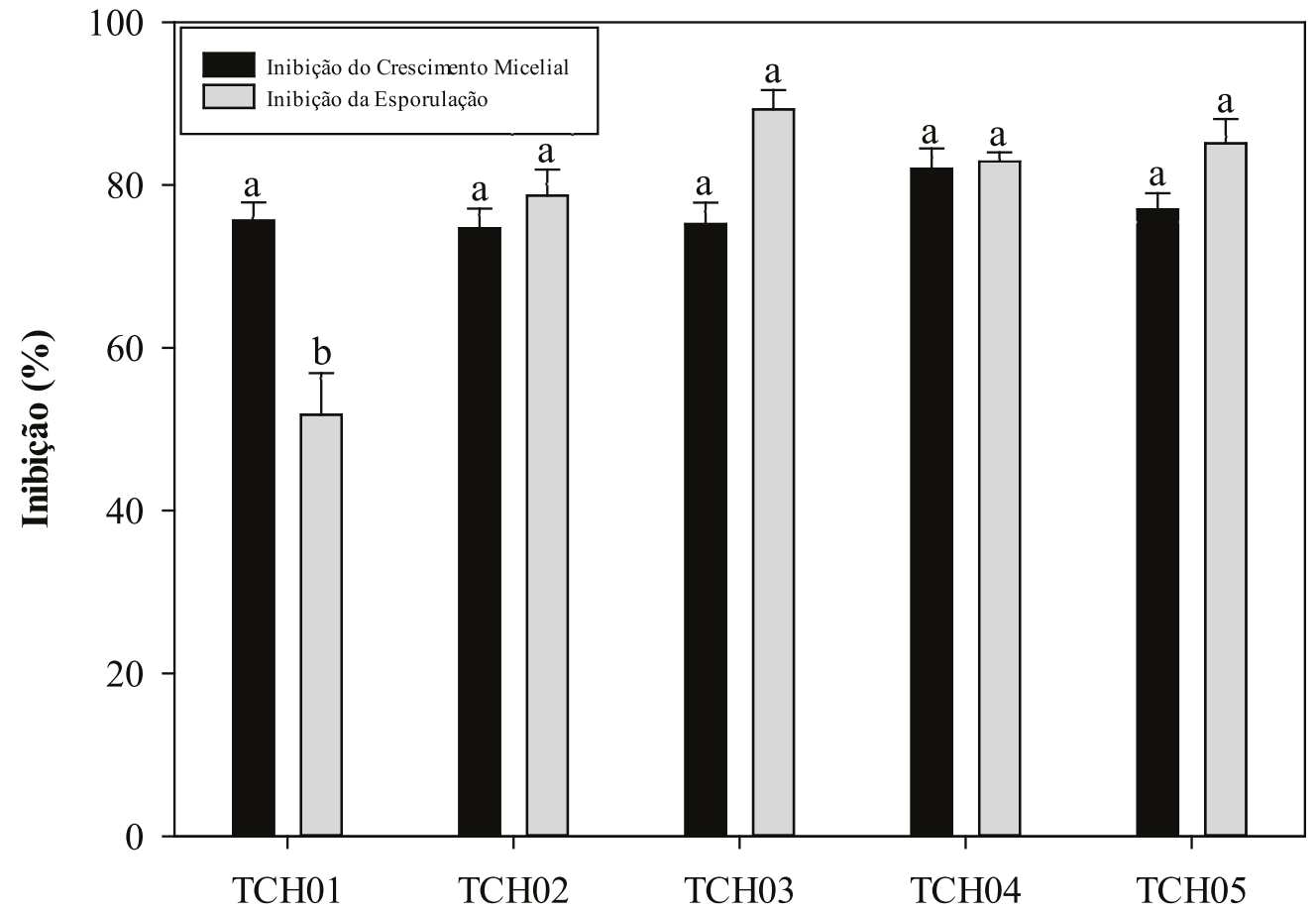

Médias com letras iguais não diferem estatisticamente entre si pelo o teste de Scott Knott $(\mathrm{p}<0,05)$.

\section{Conclusão}

Todos as cepas avaliadas apresenta potencial antagônico sobre Colletotrichum truncatum.

Evidencia-se a necessidade de testar esses isolados de Trichoderma spp. em condições de casa de vegetação e de campo, para comprovar seu potencial como agentes de biocontrole da antracnose em feijão-fava, causada por C. truncatum.

\section{Referências}

Bastos, C. N. 1997. Efeito do óleo de Piper aduncum sobre Crinipelis e outros fungos fitopatogênicos. Fitopatologia Brasileira, 22 (3): 441-443. Doi: http://dx.doi.org/10.1590/S0100-41582004000500016.

Bell, D. K.; Wells, H. D.; Markhabell, D. K.; Wells, C. R. 1982. In vitro antagonism of Trichoderma species against six fungal plant pathogens. Phytopathology, 72: 379-382. Doi: http://dx.doi.org/10.1094/ Phyto-72-379.

Bomfim, M. P; São José, A. R.; Rebouças, T. N. H.; Almeida, S. S.; Souza, I. V. B.; Dias, N. O. 2010. Avaliação antagônica in vitro e in vivo de Trichoderma spp. a Rhizopus stoloniferem no maracujazeiro amarelo. Summa Phytopathologica, 36(1): 61-67. Doi: http://dx.doi. org/10.1590/S0100-54052010000100011.

Carvalho, D. D. C.; Mello, S. C. M.; Lobo Júnior, M.; Silva, M. C. 2011. Controle de Fusarium oxysporum f.sp. phaseoli in vitro e em sementes, e promoção do crescimento inicial do feijoeiro comum por Trichoderma harzianum. Tropical Plant Pathology, 36(1): 028-034. Doi: http:// dx.doi.org/10.1590/S1982-56762011000100004.

Cavalcante, G. R. S.; Carvalho, E. M. S.; Gomes, R. L. F; Santos, A. R. B.; Santos, C. M. P. M. 2012. Reação de subamostras de feijão-fava à antracnose. Summa Phytopathologica, 38(4): 329-333. Doi: http:// dx.doi.org/10.1590/S0100-54052012000400010.
Chagas Junior, A. F; Godoy, V. H. S.; Miller, L. O.; Carvalho Filho, M. R. 2016. Bioprospecção de Trichoderma spp. sobre o crescimento micelial de Colletotrichum cliviae e Colletotrichum truncatum. Revista Brasileira de Biociências, 14(4): 238-242.

Costa, K. K.; Rufino, C. P. B.; Macedo, P. E. F.; Nogueira, S. R. 2019. Antagonismde Trichoderma spp. sobre Colletotrichum gloeosporioides, agente causal da Antracnose de Euterpe precatoria. South American Journal of Basic Education, Technical and Technological, 6(1): 391-397. https://periodicos.ufac.br/index.php/SAJEBTT/article/view/1743.

Cruz-Quiroz, R.; Roussos, S.; Rodríguez-Herrera, R.; HernandezCastillo, D.; Aguilar, C. N. 2018. Growth inhibition of Colletotrichum gloeosporioides and Phytophthora capsici by native Mexican Trichoderma strains. Karbala International Journal of Modern Science, 4(2), 237-243. Doi: https://doi.org/10.1016/j.kijoms.2018.03.002.

Daryaei, A.; Jones, E. E.; Ghazalibiglar, H.; Glare, T. R.; Falloon, R. E. 2016. Effects of temperature, light and incubation period on production, germination and bioactivity of Trichoderma atroviride. Journal of Applied Microbiology, 120(4): 999-1009. Doi: http://dx.doi.org/10.1111 / jam. 13076 .

Dennis, C.; Webster, J. 1971. Antagonistic properties of species-groups of Trichoderma. III - Hyphal interaction. Transactions of the Bristish Mycological Society, 57: 368-369. Doi: http://dx.doi.org/10.1016/ S0007-1536(71)80050-5. 
Dinesh, K.; Sinh, B.; Bai, A. T. 2018. In vitro studies on efficacy of various Trichoderma spps. Against collar rot of tomato caused by Sclerotium rolfsii Sacc. in Manipur. International Journal of Chemical Studies, 6(6), 1654-1656.

El_Komy, M. H. E.; Saleh, A. A.; Eranthodi, A.; Molan, Y. Y. 2015. Characterization of novel Trichoderma asperellum isolates to select effective biocontrol agents against tomato Fusarium wilt. The Plant Pathology Journal, 31(1): 50-60. Doi: http://dx.doi.org/10.5423 / PPJ.OA.09.2014.0087.

Fantinel, V. S.; Muniz, M. F. B.; Poletto, T.; Dutra, A. F.; Krahn, J. T.; Favaretto, R. F.; Sarzi, J. S. 2018. Biocontrole in vitro de Colletotrichum siamense utilizando Trichoderma spp. e Bacillus thuringiensis var. kurstaki. Ciência Agrícola, 16(3): 43-50. Doi: http://dx.doi.org/10.28998/rca. v16i3.4818.

Ferreira, D.F. (2010). Programa computacional Sisvar - UFLA, versão 5.4.

Henning, A. A.; Almeida, A. M. R.; Godoy, C. V.; Seixas, C. D. S.; Yorinori, J. T.; Costamilan, L. M.; Ferreira, L. P.; Meyer, M. C.; Soares, R. M.; Dias, W. P. Manual de identificação de doenças de soja. Londrina: Embrapa Soja, 5.ed, 2014, 76p.

Hillen, T.; Schwan-Estrada, K. R. F.; Mesquini, R. M.; Cruz, M. E. S.; Stangarlin, J. R.; Nozaki, M. 2012. Atividade antimicrobiana de óleos essenciais no controle de alguns fitopatógenos fúngicos in vitro e no tratamento de sementes. Revista Brasileira de Plantas Medicinais, 14(3): 439-445. Doi: http://dx.doi.org/10.1590/S1516-05722012000300003.

Lazarotto, M., Muniz, M. F. B.; Beltrame, R.; Santos, Á. F.; Müller, J.; Araújo, M. M. 2013. Tratamento biológico e químico em sementes de Cedrela fissilis para controle de Rhizoctonia sp. Cerne, 19(1): 169-175. Doi: http://dx.doi.org/10.1590/S0104-77602013000100020.

Machado, D. F. M.; Parzianello, F. R.; Silva, A. C. F.; Antoniolli, Z. I. 2012. Trichoderma no Brasil: o fungo e o bioagente. Revista de Ciências Agrárias, 35(1): 274-288.

Mbarga, J. B.; Ten hoopen, G. M.; Kuaté, J.; Adiobo, A.; Ngonkeu, M. E. L.; Ambang, Z.; Akoa, A.; Tondje, P. R.; Begoude, B. A.D. 2012. Trichoderma asperellum: A potential biocontrol agent for Pythium myriotylum, causal agent of cocoyam (Xanthosoma sagittifolium) root rot disease in Cameroon. Crop Protection, 36: 18-22. Doi: https://doi. org/10.1016/j.cropro.2012.02.004.
Nascimento, A. D.; Feijó, F. M.; Albuquerque, A. W.; Assunção, I. P.; Lima, G. S. A.; Reis, L. S. 2017. Severidade da antracnose do feijão-fava afetada por doses de cálcio e fontes de silício. Revista Ciência Agrícola, 15(2): 61-68. doi: http://dx.doi.org/10.28998/rca.v15i2.3413.

Oliveira, E. S.; Viana, F. M. P.; Martins, M. V. V. 2016. Alternativas a fungicidas sintéticos no controle da antracnose da banana. Summa Phytopathologica, 42(4): 340-350. Doi: http://dx.doi.org/10.1590/0100$5405 / 2000$.

Pereira, J. L.; Queiroz, R. M. L.; Charneau, S. O.; Felix, C. R.; Ricart, C. A. O.; Silva, F. L.; Steindorff, A. S.; Ulhoa, C. J.; Noronha, E. F. 2014. Analysis of Phaseolus vulgaris Response to Its Association with Trichoderma harzianum (ALL-42) in the Presence or Absence of the Phytopathogenic Fungi Rhizoctonia solani and Fusarium solani. Plos One, 9(5): e98234. Doi: http://dx.doi.org/10.1371/journal.pone.0098234.

Saxena, A.; Raghuwanshi, R.; Singh, H. B. 2016. Elevation of defense network in chilli against Colletotrichum capsici by phyllospheric Trichoderma strain. Journal of Plant Growth Regulation, 35(2): 377-389. Doi: https://doi.org/10.1007/s00344-015-9542-5.

Silva, J. A.; Oliveira, M. G.; Souza, L. T.; Michereff, S. J.; Assunção, I. P; Lima, G. S. A. 2014. Reação de genótipos de fava (Phaseolus lunatus L.) à podridão do colo causada por Sclerotium rolfsii. Horticultura Brasileira, 32(1): 98-101. Doi: http://dx.doi.org/10.1590/S010205362014000100016.

Vinale, F.; Sivasithamparam. K.; Ghisalberti, E. L.; Marra, R.; Woo, S. L.; Lorito, M. 2008. Trichoderma-plant-pathogen interactions. Soil Biology \& Biochemistry, 40(1): 1-10. Doi: http://dx.doi.org/10.1016/j. soilbio.2007.07.002.

Xue, A.G.; Guo, W.; Chen, Y.; Siddiqui, I.; Marchand, G.; Liu, J.; Ren, C. 2017. Effect of seed treatment with novel strains of Trichoderma spp. on establishment and yield of spring wheat. Crop Protection, 96: 97-102. Doi: http://dx.doi.org/10.1016/j.cropro.2017.02.003. 